\title{
Étude observationnelle comparative des systèmes d'agrément en Amérique du Nord et en Europe
}

\author{
Isabelle Barthélémy, Louis Rocheleau, Régis Vaillancourt et Jean-François Bussières
}

\section{INTRODUCTION}

T 'agrément (aussi appelé accréditation en Europe) est une «procédure d'évaluation externe à un établissement de santé, effectuée par des professionnels indépendants de l'établissement et de ses organismes de tutelle, évaluant l'ensemble de son fonctionnement et de ses pratiques. [II] vise à assurer la sécurité et la qualité des soins donnés au malade et à promouvoir une politique de développement continu de la qualité au sein des établissements de santé $»^{1}$.

Dans le domaine de la santé, le chirurgien Goldman entreprend, dès 1912, une évaluation de la pratique médicale américaine en commentant et publiant ses propres procédures de soins et de chirurgie. Dans la même année, l'American College of Surgeons s'inquiète du nombre croissant d'actes chirurgicaux pratiqués par les stagiaires en formation. Dans le compte-rendu de son troisième congrès annuel, l'American College of Surgeons « recommande qu'un système d'évaluation des équipements et de l'activité hospitalière soit développé afin que les institutions ayant les standards les plus élevés soient reconduites par la profession et que celles ayant de moins bons équipements et standards professionnels soient incitées à améliorer la qualité de leurs travaux. Ainsi, les patients recevront le meilleur traitement et la population aura les moyens pour identifier les meilleures institutions $»^{2}$. À la faveur de ces recommandations, le Hospital Standardization Program est lancé en $1919^{3}$. Des 692 hôpitaux visités initialement, seuls 89 satisfont aux exigences minimales de la norme $e^{4}$

Il faut attendre 1951 pour que ce programme auquel adhèrent plusieurs sociétés savantes mène à la formation de la Joint Commission for Accreditation of Hospitals. Il s'agit de la première entité indépendante et non étatique chargée de l'évaluation de la structure et de la pratique en établissement de santé sur le territoire américain. Quelques années plus tard, l'organisme étend son mandat à toutes les structures de santé, tant hospitalières qu’ambulatoires, tant publiques que privées et est renommé Joint Commission for Accreditation of Healthcare Organizations (JCAHO). Au Canada, en 1953, l'Association des hôpitaux du Canada et d'autres associations professionnelles mettent sur pied la Canadian Commission of Hospital Accreditation, qui aura pour mandat de créer un programme d'agrément. En 1958, le Conseil canadien d'agrément des hôpitaux, considéré comme l'ancêtre d'Agrément Canada, est créé. Ce dernier sera chargé d'élaborer des normes et d'évaluer leur conformité. Ce programme d'agrément est facultatif, non gouvernemental, national, bilingue et à but non lucratif. De 1960 à 1988, il gagne en popularité auprès des hôpitaux, dont le nombre d'agréés ne cesse de croître, de 350 à environ 1300. Au cours de la deuxième moitié du XXe siècle, les deux organismes américain et canadien partagent le fruit de leurs réflexions et travaux, et le cadre normatif se développe et se précise avec la publication de normes générales et spécifiques, de normes transversales et par secteur de pratique ou programmes usagers 5 . Aux États-Unis, le JCAHO est renommé The Joint Commission en $2007^{6}$. Au Canada, pour refléter l'évolution du programme et de la diversité des services évalués (notamment la psychiatrie et les soins de longue durée), l'organisme est renommé et devient le Conseil canadien des établissements de santé en 1988. Puis, toujours en raison du développement des normes, le nom est de nouveau changé en 1995 par le Conseil canadien d'agrément des services de santé puis par Agrément Canada en 20084. En 2011, le cadre normatif proposé par The Joint Commission pour le territoire américain comprend 320 normes et 1142 critères tandis que le cadre normatif proposé par Agrément Canada comprend 51 sections de normes.

En Europe, il faut attendre les années soixante-dix pour que l'évaluation des pratiques se formalise par la création d'organismes et de normes d'agrément, d'abord en GrandeBretagne puis en France ${ }^{7}$, en Suède, aux Pays-Bas, au Danemark, en Italie et en Allemagne. Il faut attendre les années quatrevingt pour que la Finlande, l'Autriche, la Hongrie, le Portugal 
et la Norvège se dotent d'initiatives similaires. ${ }^{8}$. 'International Society for Quality in Healthcare est fondée en 1985 en Italie et contribue à l'essor de ces efforts entourant l'agrément des pratiques en santé. Depuis 2002, Agrément Canada est accrédité par l'International Society for Quality in Healthcare.

En ce qui concerne la pratique pharmaceutique, il faut attendre 1936 pour que l'American College of Surgeons établisse un premier standard minimal destiné aux pharmacies d'hôpitaux. Bussières et Marando racontent que «malgré cette initiative, très peu d'hôpitaux américains emploient des pharmaciens pour prendre en charge le service de pharmacie, surtout dans les petits établissements, et ce, jusqu'à la fin des années $1950 »^{10}$. Dès sa fondation en 1942, l'American Society of Hospital Pharmacists se donne donc pour objectif de renforcer les normes minimales de la pharmacie d'hôpital afin que le service soit dirigé par un pharmacien autorisé. L'élaboration de règlements propres au service, l'aménagement d'espaces adéquats pour la préparation et la conservation des dossiers, la mise sur pied d'une bibliothèque, la conservation des médicaments et des espaces de travail réservés aux pharmaciens sont quelques-unes des autres exigences incluses dans les normes approuvées en $1950^{11}$. Ainsi, des normes spécifiques à la pratique pharmaceutique se retrouvent dans le cadre normatif du JCAHO aux États-Unis et du Conseil canadien d'agrément des services de santé au Canada, respectivement à partir de $1980^{12}$ et de $1967^{13}$. Si le cadre normatif mis en place vise avant tout l'évaluation des structures (p. ex. présence d'un local adéquat, présence de réfrigérateurs et de comptoirs de préparation appropriés), les mises à jour des années soixante-dix et quatre-vingt introduisent des critères liés au processus (p. ex. politiques et procédures de rédaction des ordonnances, politiques et procédures de déclaration d'un effet indésirable), les années quatre-vingt-dix marquent un tournant axé sur les résultats. La norme spécifique à la pharmacie est remplacée par des critères liés à la gestion optimale du médicament au sein d'autres normes liées aux usagers. Les années deux mille marquent le retour d'une norme sur la gestion des médicaments, facilitant le regroupement de plusieurs critères, sans nier l'importance de l'interdisciplinarité. Ces années sont aussi caractérisées par des normes de plus en plus orientées sur la sécurité des patients. Cela fait suite à la publication de diverses études sur les erreurs médicales au Royaume-Uni, aux ÉtatsUnis et en Australie dans le rapport de l'Institute of Medicine intitule To Err is Human : Building a Safer Health System ${ }^{14}$. Dans ces études, les événements indésirables liés aux médicaments représentent une proportion importante. Il faut également souligner l'introduction des pratiques organisationnelles requises (POR) à partir de 2005, dont une section complète concerne la gestion des médicaments. Les POR sont incluses dans la norme de gestion des médicaments à caractère hautement prioritaire. L'équivalent américain des POR sont les
National Patient Safety Goals. En 2011, le cadre normatif proposé par The Joint Commission, tout comme celui d'Agrément Canada, comprend une norme sur la gestion des médicaments.

Compte tenu de la complexité du circuit du médicament, de l'évolution de la pratique pharmaceutique et des besoins populationnels en matière de pharmacothérapie, les pharmaciens n'échappent pas à l'évaluation des pratiques. Il semble indispensable que pharmacien ait une vue d'ensemble de ce cadre d'évaluation afin de d'intervenir de façon pertinente dans cette recherche d'excellence.

\section{MÉTHODOLOGIE}

Il s'agit d'une étude observationnelle comparative de la structure et des pratiques d'agrément des établissements de santé. L'objectif principal consiste à comparer ces organismes en Europe et en Amérique du Nord. L'étude met en évidence deux organismes nord-américains, la Joint Commission aux ÉtatsUnis et Agrément Canada au Canada, ainsi que 20 organismes européens. L'objectif secondaire vise à comparer les normes relatives au circuit du médicament et à la pratique pharmaceutique en France, aux États-Unis et au Canada.

En ce qui concerne l'objectif primaire, nous avons relevé 65 variables afin de comparer les organismes d'agrément au Canada, aux États-Unis et en Europe. Ces variables concernaient l'identification, la politique, la gestion, les standards, les enquêteurs, l'évaluation, les récompenses, la finance et l'information et ont été choisies à partir des données disponibles dans une enquête européenne publiée en $2008^{15}$. Cette étude européenne avait été menée par le Centre fédéral d'expertise des soins de santé et avait comme objectif d'établir les domaines d'agrément propices pour le système de santé belge. Dans cette enquête européenne, les auteurs proposent une revue des mécanismes d'agrément par pays et des organismes en place ou en développement. Nous avons ensuite tenté d'apparier les 65 variables de l'enquête européenne aux données disponibles sur les sites Internet d'Agrément Canada et de la Joint Commission. Il n'y a pas eu de vérification téléphonique, par courriel ou autrement auprès de la Joint Commission; des clarifications verbales ont été obtenues auprès d'Agrément Canada compte tenu qu'un des auteurs est aussi visiteur. Vingt-quatre variables ont pu être préalablement appariées à la suite d'une saisie de données par deux assistants de recherche dans un tableau comparatif en 2010. À la suite d'une mise à jour de la comparaison en 2011, 11 variables comportant suffisamment de renseignements ont été retenues pour la comparaison et sont présentées au tableau 1 . L'utilisation des données de l'enquête européenne a fait l'objet d'une autorisation d'utilisation auprès de l'équipe de recherche. À noter que le nombre de pays pour lequel nous disposons de données varie selon les variables comparées, compte tenu des données recueillies dans l'enquête européenne. 
Tableau 1. Profil comparatif de l'agrément en Europe et en Amérique du Nord

Variable appariée avec les données nord-américaines*

Europe (pays)

Amérique du Nord

Par quels modèles les états ont-ils été inspirés pour

définir leurs normes d'agrément dans le domaine

de la santé? $(n=21)$

European Foundation for Quality Management International Organization for Standardization

$E C, F I, H L, I T, L U-I(5)$

Joint Commission on Accreditation of Healthcare Organizations,

Canadian Council on Health Services Accreditation

Australian Council on Healthcare Standards

Quel est le statut légal de l'organisme d'agrément? $(n=22)$

Une agence gouvernementale

$\mathrm{BG}, \mathrm{FI}(2)$

$A L, E S, F I, F R, H L, I E, I T, P L, R T(9) \quad$ Canada, ÉUA

Une agence non gouvernementale à but non lucratif

$\mathrm{AL}, \mathrm{Fl}, \mathrm{FR}(3)$

Une entité à but lucratif

Quel lien existe-t-il entre l'organisme d'agrément

et le Gouvernement? $(\boldsymbol{n}=\mathbf{2 2})$

L'organisme est géré par le ministère de la santé

L'organisme est une agence gouvernementale indépendante

L'organisme est une agence indépendante représentée au sein

du gouvernement

L'organisme est indépendant sans lien formel avec le gouvernement

BG, ES-A, ES-C, FR, IE, IT, LE, LU-A, PL, RU-HCC (10)

$A L, E C, E S, E S-V, H L, L U-I, P T(7) \quad$ Canada, ÉUA

$\mathrm{FI}, \mathrm{RT}, \mathrm{RU}-\mathrm{HAQU}(3)$

La visite d'agrément est-elle une exigence légale ou une

démarche volontaire de la part des hôpitaux? $(n=22)$

Exigence légale

EC, ES-C, LU-A, PT, RU-HCC (5)

$F R$, IT, LE, PL (4)

$B G, E S-A, E S-V, I E, R U-H A Q U$ (5) ÉUA

$\mathrm{AL}, \mathrm{ES}, \mathrm{FI}, \mathrm{HL}, \mathrm{LU}-\mathrm{I}, \mathrm{RT}(6)$

Canada

$B G, E C, E S-A, E S-C, F R, ~ I T, L E$, LU-A RU-HCC (9)

Démarche volontaire RU-HAQU (11)

Canada (mais exigée au Québec), ÉUA

La décision d'attribution ou non de l'agrément est-elle binaire ou comporte-t-elle plusieurs mentions? $(n=12)$

Binaire : Accrédité/Non accrédité

Avec mentions

Pour combien d'années au maximum la décision d'attribution

d'agrément est-elle valide? $(n=25)$

1 an

3 ans

4 ans

ES, ES-V, FI, IE, IT, PL, PT, RT, RU-HAQU (9) Canada, ÉUA

$B G, F R, H L(3)$

Possiblement au Québec avec une modification de la LSSSS (Loi sur les services de santé et les services sociaux)

5 ans

ES, ES-V, FI, HL, IE, LU-A, PL, PT, RT,

RU-HAQU (10)

Les résultats détaillés de la visite de l'hôpital sont-ils diffusés sur Internet? $(n=12)$

Oui

Non

FR, RU-HCC (2)

EC, ES-A, ES-V, FI, HL, IE, LE, RT (8)

ÉUA

Canada

Quel est le coût annuel du processus d'agrément pour un

hôpital de 100 lits ? $(n=17)$

Gratuit

$100 \$$ à $1000 \$$

$1000 \$$ à $10000 \$$

$10000 \$$ à $100000 \$$

$>100000 \$$
EC (1)

LE (1)

BG, LU-A, LU-I , PL, RT (5)

ES-A, ES-V, HL, PT (4)

FR, IE, RU-HAQU, RU-HCC (4)
Canada, ÉUA 
Tableau 1. Profil comparatif de l'agrément en Europe et en Amérique du Nord (suite et fin)

Variable appariée avec les données nord-américaines*

Europe (pays)

Amérique du

Nord

Quelle est la composition du conseil d'administration

de l'organisme? $(n=22) \dagger$

Professionnels de la santé en exercice

$A L, E C, E S, E S-A, E S-V, F I, F R, H L, I E, L U-I, \quad C a n a d a, E ́ U A$

PT, PL, RU-HAQU (13)

Représentants des autorités réglementaires du pays

ES, ES-A, ES-C, FR, IT, LU-A, PL (7)

Canada, ÉUA

$A L, B G, E S-A, F I, F R, I E, R T(7)$

Canada

Représentants d'établissement de santé

$E C, E S-V, F R, H L, I E, R U-H C C(6)$

ÉUA

Représentants d'établissements d'enseignement

Tiers-payeurs

ES, IE, LU-I, PL, RU-HAQU (5)

Canada

Aucun représentant externe

FR, HL, LU-I (3)

Quelle est la composition de l'équipe de visiteurs? $(n=17) \dagger$

Membres de la direction

LE (1)

Médecins

AL, BG, ES, ES-A, ES-V, FI, FR, HL, IE, LE, Canada, ÉUA

LU-I, PT, PL, RT, RU-HAQU (15)

$A L, B G, E S, E S-A, E S-V, F I, F R, H L, I E$, LE, Canada, ÉUA

LU-I, PL, PT, RT, RU-HAQU (15)

Infirmières

$A L, B G, E S, E S-A, E S-V, F I, F R, H L$, IE, LU-I,

Canada, ÉUA

$\mathrm{PL}, \mathrm{PT}, \mathrm{RT}, \mathrm{RU}-\mathrm{HAQU}(14)$

Pharmaciens

Non-précis

Canada (4 \%

du personnel

de visiteur ${ }^{16}$ )

Quel est le concept retenu pour décrire le cadre normatif

utilisé par l'organisme externe? $(n=14)$

Niveaux minimums exigés

Cibles d'excellence

Niveaux minimums et cibles d'excellence

BG, LE (2)

EC, ES, ES-V, FR, IE, PT (6)

ES-A, HL, RU-HAQU, RU-HCC (4)

Canada, ÉUA

$\mathrm{AL}=$ Allemagne, $\mathrm{BG}=$ Bulgarie, $\mathrm{EC}=$ Écosse, $\mathrm{ES}=$ Espagne, $\mathrm{ES}-\mathrm{A}=$ Espagne (Andalousie), $\mathrm{ES}-\mathrm{C}=$ Espagne (Catalogne),

$\mathrm{ES}-\mathrm{V}=$ Espagne (Valence), ÉUA = États-Unis d'Amérique, $\mathrm{FI}=$ Finlande, $\mathrm{FR}=$ France, $\mathrm{HL}=\mathrm{Hollande}$, IE = Irlande, IT = Italie

(La Marche), LE = Lettonie, LU-A = Luxemburg : exploitation Autorisation, LU-I = Luxemburg : qualities Incentives, PL = Pologne,

PT = Portugal, RT = République Tchèque, RU = Royaume Uni, RU-HAQU = Healthcare Accreditation \& Quality Unit,

RU-HCC $=$ HealthCare Commission .

${ }^{*} \mathrm{n}=$ nombre de pays représentés dans la réponse à une question, y compris les organismes nord-américains. Le nombre total de

pays applicables par question peut varier selon les résultats de l'enquête européenne.

†À noter que dans certaines questions, un pays peut répondre à plusieurs options

En ce qui concerne l'objectif secondaire, nous avons consulté les sites Internet des organismes français, américain et canadien afin de comparer l'encadrement normatif lié au circuit du médicament et à la pratique pharmaceutique. Aucune analyse statistique n’a été effectuée.

\section{RÉSULTATS}

À partir de l'enquête européenne publiée, nous avons retenu 11 variables aux fins de comparaison avec les sites web d'Agrément Canada ${ }^{17}$ et de la Joint Commission ${ }^{18}$ consultés au cours de l'année 2010 et en novembre 2011. Notre étude observationnelle comparative met en évidence la présence d'un programme structuré d'agrément des hôpitaux et des structures de santé dans $52 \%$ des pays européens et dans les deux pays nord-américains. Le tableau 1 présente les éléments de comparaison. Afin de faciliter la comparaison, nous avons formulé des questions simples permettant de présenter les résultats issus de notre analyse.
Le tableau 2 présente une comparaison des organismes d'agrément et de l'encadrement normatif de la gestion des médicaments et de la pratique pharmaceutique en France, aux États-Unis et au Canada. Dans le cas des États-Unis, nous avons retenu pour les fins de la comparaison les normes de la filiale internationale de la Joint Commission, soit la Joint Commission International. Notons également que, contrairement à l'Amérique du Nord, les normes relatives à la gestion du médicament en France ne font pas l'objet d'une section à part entière dans le manuel de certification des établissements de santé.

\section{DISCUSSION}

Cette étude observationnelle met en évidence le développement d'organismes d'agrément et de processus d'évaluation de plus en plus structurés dans le domaine de la santé à l'échelle européenne et nord-américaine. Bien que seules 11 des 65 variables de l'enquête européenne aient pu être 
Tableau 2. Comparaison des normes sur la gestion des médicaments et de la pratique pharmaceutique en France, aux États-Unis et au Canada

Variable

France

États-Unis*

Canada

Nom de Haute autorité de santé Joint Commission International Agrément Canada

l'organisme

Site web www.has-sante.fr/ www.jointcommission www.accreditation.ca/fr

Programme Certification des international.org/ d'agrément établissements de santé Nom des outils Manuel de certification des et version établissements de santé (version 2010)

Structure normative Deux sections, soit la gestion globale de l'établissement de santé et la prise en charge du patient Accreditation for hospitals Programme Qmentum Accreditation Standards for Hospitals (1 ${ }^{\text {er }}$ janvier 2011) Normes disponibles sur le site web; les versions varient selon la date de dernière mise à jour Deux sections, soit la gestion de l'établissement de santé Une norme sur la gouvernance, une norme sur (six sous-sections) et la prise en transversales (c.-à-d. gestion des médicaments charge des patients (huit sous- et prévention et contrôle des infections), six sections) normes populationnelles (p. ex. mère-enfant, cancer, maladie chronique, maladie mentale, enfants et jeunes, santé publique), 38 normes d'excellence des services (p. ex. urgence, ambulatoire, soins intensifs) et trois normes de distinction.

\begin{tabular}{llll}
\hline Contenu normatif & 8 thématiques & 7 thématiques & 7 thématiques \\
relatif à la gestion & 28 normes & 21 normes & 22 normes \\
du médicament & 85 critères & 84 critères & 135 critères
\end{tabular}

Libellé des 1 ) Gestion de la qualité et de la 1) Organization and thématiques relatives à la gestion des médicaments

1) Gestion de la qualite
sécurité des soins

- Programme global et coordonnées de gestion de la qualité et de la qualité des soins

2) Droit et place des patients

- Prise en charge de la douleur

3) Parcours du patient

- Prise en charge médicamenteuse

- Éducation thérapeutique destinée au patient et à son entourage management: medication

use in the organization complies with applicable laws and regulations and is organized to meet patient needs

2) Selection and procurement: an appropriate selection of medications for prescribing or ordering is stocked or readily available

3) Storage: medications are properly and safely stored

4) Ordering and transcribing: prescribing, ordering, and transcribing are guided by policies and procedures

5) Preparing and dispensing: medications are prepared and dispensed in a safe and clean environment

6) Administration: medications effects on patients are monitored

7) Monitoring: medication effects on patients are monitored
1) Travailler ensemble à promouvoir la sécurité liée aux médicaments

2) Sélectionner et se procurer les médicaments avec soin

3) Étiqueter et entreposer les médicaments de façon appropriée

4) Commander et transcrire les médicaments de façon appropriée

5) Préparer et délivrer les médicaments avec exactitude

6) Administrer les médicaments aux clients de façon sécuritaire

7) Évaluer la qualité et obtenir des résultats positifs

\section{Nombre}

5

9

d'indicateurs

impliquant la

gestion des

médicaments 


\section{Tableau 2. Comparaison des normes sur la gestion des médicaments et de la pratique pharmaceutique en France, aux États-Unis et au Canada (suite)}

Variable

Intitulé des indicateurs impliquant la gestion des médicaments
France

1) Bon usage des antibiotiques 1) Processes: selection,

2) Gestion du dossier du patient

3) Prise en charge de la douleur

4) Prise en charge médicamenteuse du patient

5) Troubles de l'état nutritionnel procurement, storage securing, prescribing and ordering, transcribing, preparing, dispensing, administration monitoring

2) Patient safety goals

3) Errors

4) Education

5) Information management

6) Evaluation

7) Risks

8) Medication reconciliation

9) Patient involvement

Évaluation des Aucune donnée disponible indicateurs sur le site Internet

\section{Canada}

Le bilan comparatif des médicaments à

l'admission
6 thématiques incluant au total 47 cas indicateurs :

1) Hospital and critical access hospital

2) Ambulatory care and office based surgery

3) Behavioural health care

4) Home care

5) Long-term care

6) International
Les outils sont des questionnaires au moyen desquels on recueille l'information sur des éléments essentiels des services de santé. Nous comptons trois outils dans le cadre du programme Qmentum :

1) L'outil d'évaluation du fonctionnement de la gouvernance, rempli par les membres du conseil d'administration;

2) Le sondage sur la culture de sécurité des patients, rempli par les membres du personnel;

3) Le sondage Pulse sur la qualité de vie au travail, rempli par les membres du personnel.

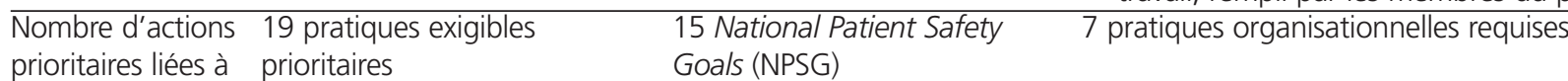

la gestion des

médicaments

Intitulé des Selon le libellé « Mettre en

actions prioritaires œuvre »

impliquant la

gestion des

- Critère 12.a - des formations/ actions sont mises en œuvre dans les secteurs d'activité; les secteurs d'activité mettent en place l'éducation du patient à la prise en charge de la douleur; la traçabilité des évaluations de la douleur dans le dossier du patient est assurée; les professionnels de la santé s'assurent du soulagement de la douleur; des moyens d'évaluation de la douleur pour les patients non communicants sont mis à la disposition du personnel

- Critère 20.a - des actions de sensibilisation et de formation des professionnels au risque d'erreurs médicamenteuses sont menées ;

l'informatisation de la prise en charge médicamenteuse est engagée.
15 National Pati
Goals (NPSG)

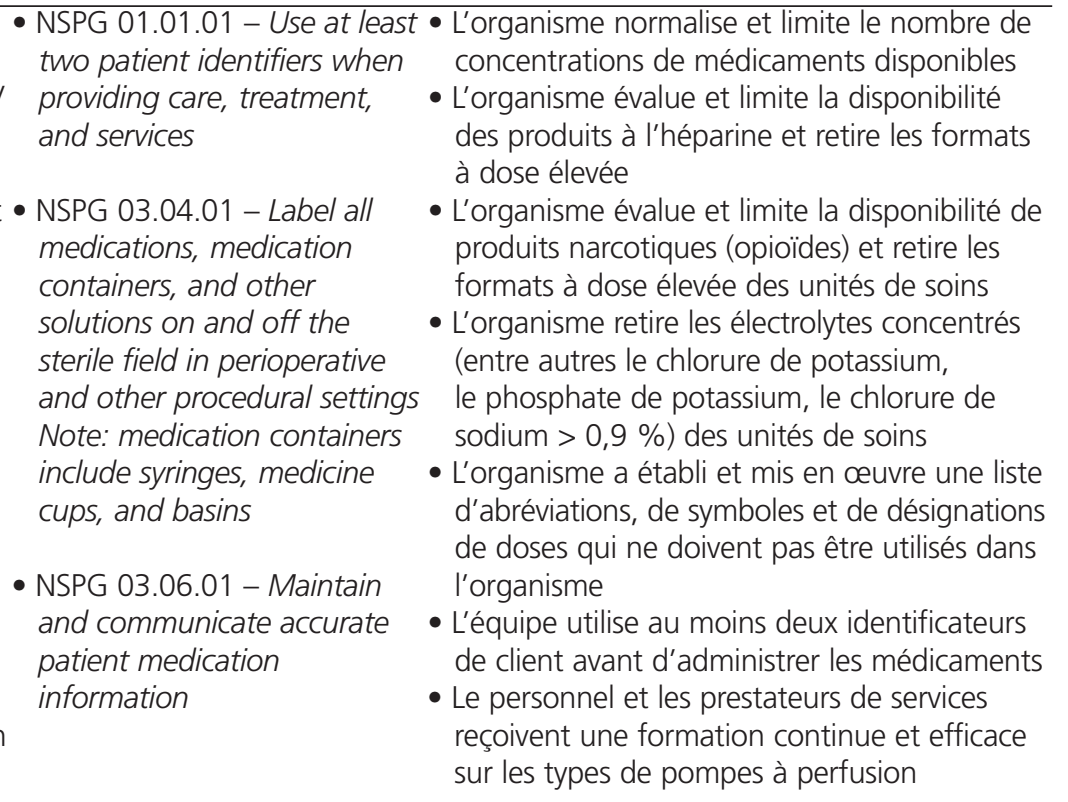


Tableau 2. Comparaison des normes sur la gestion des médicaments et de la pratique pharmaceutique en France, aux États-Unis et au Canada (suite et fin)

Variable

France

États-Unis*

Canada

- Critère 20.a bis : les règles de

prescription sont mises en

œuvre ; le développement

de l'analyse pharmaceutique

des prescriptions et de la

délivrance nominative des

médicaments est engagé ;

les bonnes pratiques de

préparation sont appliquées ;

les professionnels de la santé

assurent l'information des

patients sur le bon usage des

médicaments ; la traçabilité

de l'administration des

médicaments dans le dossier

du patient est assurée.

- Critère 23.a : selon la nature

des besoins et des attentes

des patients, des programmes

d'éducation thérapeutique du

patient autorisés sont proposés

aux patients et mis en œuvre ;

l'établissement définit dans

son plan de formation une

formation pour l'éducation

thérapeutique du patient.

- Critère 26 (a et b) : la

traçabilité des dispositifs

médicaux implantables

incluant les vérifications

effectuées, est assurée

- Critère 28.a : les différents

secteurs d'activité clinique et

médico-technique mettent en

œuvre des démarches d'EPP ;

l'engagement des

professionnels est effectif ;

des actions de communication

relatives aux démarches d'EPP

et à leurs résultats sont mises

en œuvre

${ }^{\star} \grave{A}$ noter que le libellé anglais a été retenu lorsqu'il n'y avait pas de traduction officielle disponible.

appariées, la comparaison met en évidence quelques éléments dignes d'intérêt. Il est intéressant de constater que l'approche nord-américaine a inspiré le développement d'un processus similaire dans plusieurs pays européens. De plus, au moins neuf pays européens ont fait de l'obtention d'un agrément une obligation légale. Au Canada, cette obligation pour les établissements de santé n'est actuellement en vigueur qu'au Québec. Seuls deux pays européens rendent publics les résultats détaillés du rapport d'agrément. Bien que ce nombre soit limité, il est raisonnable de penser que cette tendance pourrait être à la hausse compte tenu des sommes investies dans les systèmes de santé à travers le monde. On dispose malheureusement de trop peu de données pour confirmer la présence ou l'absence de pharmaciens au processus de visite d'agrément. Dans les éléments clés mis en évidence dans l'enquête européenne, les auteurs soulignent qu'il n'y a pas encore de preuve d'une relation positive de cause à effet entre les résultats d'une visite d'agrément et les résultats de santé observés auprès d'une patientèle traitée au sein de l'établissement visité. Les exercices d'agrément reposent encore trop souvent sur l'évaluation de structures et de processus et pas assez sur des marqueurs de résultats cliniques. Ce propos nous rappelle 
l'importance de nous intéresser au cadre normatif, de le commenter lorsque les organismes d'agrément le permettent et de nous approprier les normes pour qu'elles aient un effet réel au quotidien.

En ce qui concerne le circuit du médicament, l'évolution du cadre normatif de ces organismes met en évidence un intérêt croissant pour ce circuit, compte tenu de sa complexité et de ses conséquences sur les résultats de santé. Toutefois, notre comparaison des normes proposées en France, aux États-Unis et au Canada met en évidence des différences importantes portant sur les thèmes retenus, le nombre de normes et le nombre de critères. Alemanni et coll. ont réalisé une comparaison de la version 2008 de la norme sur la gestion des médicaments aux États-Unis et au Canada ${ }^{19}$. Cette comparaison a mis en évidence un total de 143 normes canadiennes et de 103 normes américaines. Fait intéressant, $43 \%$ des 143 critères canadiens ont pu être appariés directement à un critère américain alors que $68 \%$ des 103 critères américains ont pu être appariés à au moins un critère canadien. Six des critères américains ont été appariés à plus d'un critère canadien et 12 critères canadiens ont pu être appariés à plus d'un critère américain. Quatre des 22 normes canadiennes n'avaient aucun critère équivalant aux normes américaines. Plusieurs facteurs peuvent expliquer ces différences, notamment le cadre législatif en vigueur, les experts consultés pour la rédaction des normes, la formation respective et le rôle des pharmaciens au sein de chaque pays. Si le circuit du médicament fait clairement l'objet d'un intérêt sans précédent en disposant de normes et de critères spécifiques auprès de ces organismes d'agrément, d'autres autorités réagissent en proposant des cadres normatifs complémentaires. Au Québec, par exemple, l'Ordre des pharmaciens du Québec publiait en 2010 ses premiers standards de pratique ${ }^{20}$, l'Association paritaire pour la santé et la sécurité au travail du secteur affaires sociales publiait en 2008 son guide de prévention de la manipulation des médicaments dangereux ${ }^{21}$, le ministère de la Santé et des Services sociaux publiait en 2005, puis en 2008 et en 2011 ses critères relatifs au dossier sur les systèmes automatisés et robotisés de distribution des médicaments ${ }^{22}$ sans compter les deux nouvelles normes sur les préparations stériles et non stériles de l'Ordre des pharmaciens du Québec à paraître en $2012^{23}$. Soulignons également que, dans le cadre de la campagne nationale pour des soins plus sécuritaires, certains organismes, comme l'Institut canadien pour la sécurité des patients, ont participé à la promotion du bilan comparatif des médicaments à l'admission ${ }^{24}$.

Notre revue documentaire met aussi en évidence le fait que le circuit du médicament n'est plus l'affaire du seul pharmacien. Si le cadre normatif nord-américain avait pour cible la pharmacie au cours des années soixante à quatre-vingt-dix, les nouvelles normes ciblent le médicament, son usage et les pratiques professionnelles, y compris l'exercice de la pharmacie, peu importe le lieu de prestation des services au sein de l'établissement. Il faut toutefois reconnaître beaucoup moins de précision quant au rôle du pharmacien dans le cadre normatif français, conséquence de la dotation plus limitée de pharmaciens en établissement de santé et de leur rôle clinique émergent. En outre, ce foisonnement de normes et de critères s'accompagne de priorités, nommées "pratiques exigibles prioritaires " en France, National Patient Safety Goals aux États-Unis et " pratiques organisationnelles requises » au Canada. Ce concept peut aider les pharmaciens à prioriser leurs actions.

À la lumière de ce portrait du cadre normatif, que faut-il retenir? Nous pensons que les pharmaciens ont intérêt à discuter des pratiques d'évaluation avec des collègues d'autres pays, notamment lors de la participation à des congrès à l'extérieur du Canada. En évaluant la documentation internationale, les pharmaciens devraient davantage s'intéresser aux cibles d'excellence mises en évidence par ces normes d'agrément. En préparant la planification stratégique de leur département de pharmacie, les pharmaciens doivent envisager un outil pratique de gestion de tous ces critères afin d'avoir une vue d'ensemble cohérente en appariant notamment les critères similaires et en cotant périodiquement le degré de conformité locale. Au CHU Sainte-Justine, nous prévoyons que cette réflexion se traduira par un tableau de bord unique, intégrant et regroupant tous ces critères, avec des filtres web permettant un affichage des critères non conformes par secteur de pratique. Lorsque son développement sera terminé, nous prévoyons offrir l'outil développé en ligne à tout département de pharmacie canadien qui souhaite développer une vue d'ensemble du cadre normatif applicable en pharmacie et compiler son propre état de conformité.

Si les auteurs de l'enquête européenne considèrent, en observant leur revue documentaire, qu'il n'y a pas encore de preuve d'une relation positive de cause à effet entre les résultats d'une visite d'agrément et les résultats sur la santé observés auprès des patients traités au sein de l'établissement visité, les pharmaciens ne doivent pas perdre de vue l'importance de cibler leurs efforts sur des actions pouvant avoir des retombées maximales sur la qualité des soins et la réduction des risques. À cette fin, les pharmaciens doivent participer activement aux rétroactions demandées par Agrément Canada, lors de la révision du cadre normatif, afin d'assurer un développement cohérent de la norme. Agrément Canada reverra en 2012 la norme sur la gestion des médicaments.

Cette étude observationnelle comporte des limites. Les données relatives aux organismes français, américain et canadien ont été recueillies uniquement à partir de sites Internet. Les auteurs de cette étude connaissent davantage le cadre normatif canadien que les deux autres, compte tenu de leur participation au processus de visite ou comme établissement visité. 


\section{CONCLUSION}

Il existe peu de données comparatives portant sur la structure et les pratiques d'agrément des établissements de santé. Cette étude observationnelle compare la situation au Canada, aux États-Unis et en Europe. L'étude met en évidence deux organismes nord-américains, la Joint Commission aux ÉtatsUnis et Agrément Canada au Canada, ainsi que 20 organismes européens. De plus, l'étude met en évidence le cadre normatif relatif au circuit du médicament et à la pratique pharmaceutique aux États-Unis, au Canada et en France.

\section{Références}

1. Caducée. Accréditation : quelques définitions. Cenon (France) : Caremedia. Publié au www.caducee.net/Droit-Sante/Veille/accreditation.asp. Consulté le 28 octobre 2011.

2. Histoire de l'évaluation et de la qualité des soins. Oran (Algeria) : Agence Nationale pour le Développement de la Recherche en Santé, Laboratoire de la qualité des soins. Publié au www.ands.dz/qualitelab/SITE/Lab.Audit/A0013.pdf. Consulté le 28 décembre 2011.

3. The 1919 "minimum standard" document. Chicago (IL) : American College of Surgeons. Publié au www.facs.org/archives/minimumhighlight. html. Consulté le 19 janvier 2012.

4. Historique (1918). Ottawa (ON) : Agrément Canada. Publié au www.accreditation.ca/a-propos/historique/. Consulté le 27 décembre 2011.

5. Shaw CD. External assessment of health care. BMJ 2001;322(7290): 851-854.

6. The Joint Commission history. Deer Park (IL) : The Joint Commission. Publié au www.jointcommission.org/assets/1/18/ joint_commission_ history_20111.pdf. Consulté le 29 décembre 2011.

7. Certification des établissements de santé. Saint-Denis La Plaine (France) : Haute autorité de santé. Publié au www.has-sante.fr/portail/jcms/c_5076/ certification-des-etablissements-de-sante?cid=c_5076. Consulté le 28 décembre 2011.

8. Historique de l'organisation de l'accréditation. Paris (France) : Techniques de l'ingénieur. Publié au www.techniques-ingenieur.fr/base-documentaire/ mesures-analyses-th1/qualite-au-laboratoire-42497210/evaluation-de-lacompetence-des-laboratoires-sl1610/bref-historique-de-l-organisation-de1-accreditation-s11610niv10002.html. Consulté le 28 décembre 2011.

9. Shaw CD. Toolkit for accreditation programmes: some issues in the design and redesign of external health care assessment and improvement systems. Melbourne (Australia) : International Society for Quality in Health Care; 2004. Publié au www.hciproject.org/sites/default/files/Toolkit_for_ Accreditation_Programs_ISQua.pdf. Consulté le 28 décembre 2011.

10. Bussières JF, Marando N. De l'apothicaire au spécialiste. Histoire de la pharmacie hospitalière au Québec. Montréal (QC) : Association des pharmaciens des établissements de santé du Québec; 2011. Publié au www.apesquebec.org/apothicaire. Consulté le 27 décembre 2011.

11. Harris RR, McConnell WE. The American Society of Hospital Pharmacists: a history. Am J Hosp Pharm 1993;50 Suppl 2:S3-S45.

12. Hoffmann RP. The six major JCAHO pharmacy standards. Hosp Pharm 1988;23(11):988, 990.

13. Compendium des guides pour l'accréditation des hôpitaux. Toronto $(\mathrm{ON})$ : Conseil canadien d'accréditation des hôpitaux; 1967.

14. Kohn LT, Corrigan JM, Donaldson MS, editors. To err is human: building a safer health system. Washington (DC): National Academy Press, Institute of Medicine; 1999. Publié au www.nap.edu/openbook. php?record_id=9728. Consulté le 3janvier 2012.

15. Walcque CD, Seutjens B, Vermeyen K, Peeters G, Vinck I. Étude comparative des programmes d'accréditation hospitalière en Europe. KCE Report 70B, D/2008/10.273/02. Bruxelles: Centre fédéral d'expertise des soins de santé; 2008. Publié au http://kce.fgov.be/sites/default/files/ page_documents/d20081027302.pdf. Consulté le 3 octobre 2011.

16. Rapport annuel 2010. Ottawa (ON) : Agrément Canada; 2010. Publié au www.accreditation.ca/uploadedFiles/Rapport\%20annuel\%202010.pdf. Consulté le 3 janvier 2012.
17. Page d'accueil. Ottawa (ON) : Agrément Canada. Publié au www. accreditation.ca/fr/Default.aspx?rdr=true\&LangType=3084. Consulté le 3 janvier 2012

18. Home page. Oakbrook Terrace (IL) : The Joint Commission. Publié au www.jointcommission.org/. Consulté le 3 janvier 2012.

19. Alemanni J, Brisseau L, Lebel D, Vaillancourt R, Rocheleau L, Bussières JF. Comparative study of the clarity and assessability of the drug management standards of Accreditation Canada and the US Joint Commission. Can J Hosp Pharm 2011;64(2):116-123.

20. Standards de pratique. Montréal (QC) : Ordre des pharmaciens du Québec; 2010. Publié au www.opq.org. Consulté le 29 décembre 2011.

21. Guide pour la prévention de la manipulation des médicaments dangereux. Association pour la santé et la sécurité au travail, Secteur des affaires sociales; 2008. Publié au www.asstsas.qc.ca/publications/publicationsspecialisees/guides-de-prevention/guide-de-prevention-manipulationsecuritaire-des-medicaments-dangereux.html. Consulté le 2 décembre 2011.

22. Guide logistique hospitalière 2011. Québec (QC) : Corporation d'hébergement du Québec; 2011. Publié au www.msss.gouv. qc.cal documentation/planification-immobiliere/app/DocRepository/1/ Publications/Guide/110629_Guide_logistique_hospitaliere.pdf. Consulté le 3 janvier 2012.

23. Rapport annuel 2010-2011. Montréal (QC) : Ordre des pharmaciens du Québec. Publié au www.opq.org/fr/media/docs/rapport_annuel_20102011.pdf. Consulté le 4 janvier 2012.

24. Bilan comparatif des médicaments. Edmonton (AB) : Canadian Patient Safety Institute; 2012. Publié au www.saferhealthcarenow.ca/FR/ Interventions/medrec/Pages/default.aspx. Consulté le 3 janvier 2012.

Isabelle Barthélémy est interne en pharmacie, Département de pharmacie et Unité de recherche en pratique pharmaceutique, $\mathrm{CHU}$ Sainte-Justine, Montréal, Québec. Elle est aussi étudiante en pharmacie à la Faculté de pharmacie, Université de Lyon, Lyon, France.

Louis Rocheleau, MA, est Coordonnateur intérimaire à la gestion de la qualité et des risques, Direction des risques et de la qualité, $\mathrm{CHU}$ Sainte-Justine, Montréal, Québec.

Régis Vaillancourt, B.Pharm., Pharm.D., FCSHP, est chef, Département de Pharmacie, Centre hospitalier pour enfant de l'est de I'Ontario, Ottawa. II est également rédacteur adjoint du JCPH.

Jean-François Bussières, B. Pharm., M. Sc., FCSHP, est chef, Département de pharmacie et Unité de recherche en pratique pharmaceutique, CHU Sainte-Justine, et professeur titulaire de clinique, Faculté de pharmacie, Université de Montréal, Montréal, Québec.

\section{Adresse de correspondance :}

Jean-François Bussières

Département de pharmacie

Centre Hospitalier Universitaire Sainte-Justine

3175, chemin de la Côte Sainte-Catherine

Montréal QC H3T 1C5

Courriel : jf.bussieres@ssss.gouv.qc.ca

\section{Remerciements}

Nous remericons Jordane Alemanni, interne à I'Université de Toulouse III, Paul Sabatier, Toulouse, France, et Lionel Brisseau, interne à I'Université de Nantes, Nantes, France, pour la saisie de données initiale. Jordane Alemanni et Lionel Brisseau sont aussi assistants de recherche à I'Unité de recherche en pratique pharmaceutique, CHU Sainte-Justine, Montréal, Québec. 